Network Working Group

Request for Comments: 4024

Category: Informational
G. Parsons Nortel Networks

J. Maruszak July 2005

\title{
Voice Messaging Client Behaviour
}

Status of This Memo

This memo provides information for the Internet community. It does not specify an Internet standard of any kind. Distribution of this memo is unlimited.

Copyright Notice

Copyright (C) The Internet Society (2005).

Abstract

This document defines the expected behaviour of a client to various aspects of a Voice Profile for Internet Mail (VPIM) message or any voice and/or fax message.

Table of Contents

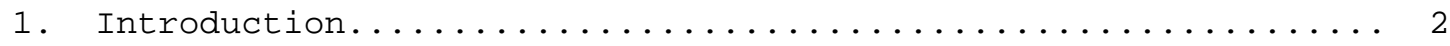

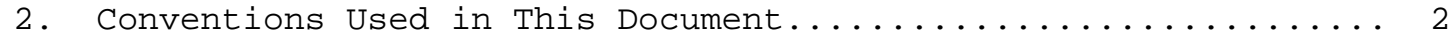

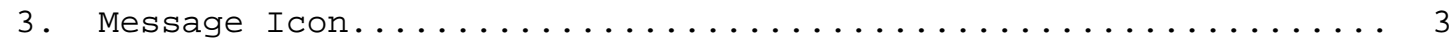

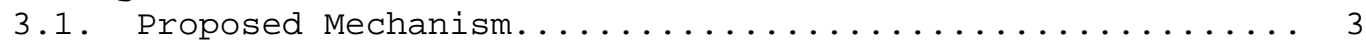

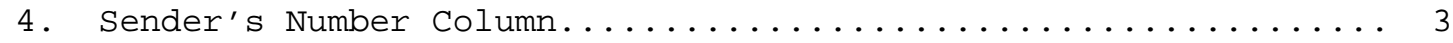

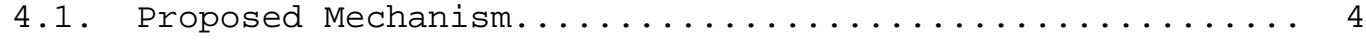

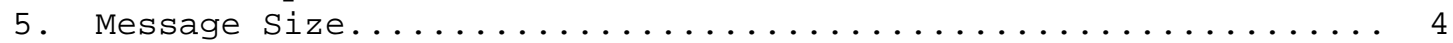

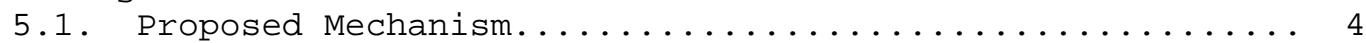

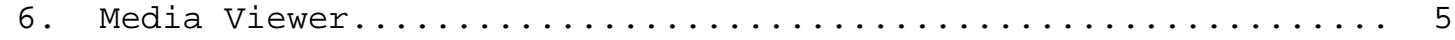

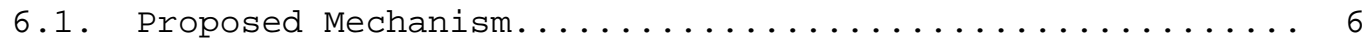

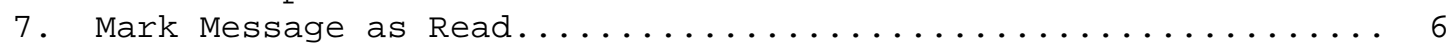

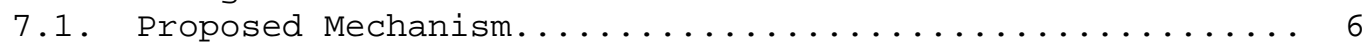

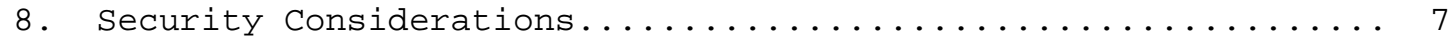

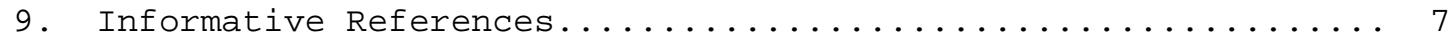

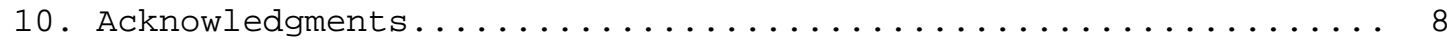




\section{Introduction}

As Internet messaging evolves into unified messaging, the term "e-mail" no longer refers to text-only messages. Today's "e-mail" are often multi-media. That is, they can have numerous non-text parts. These parts can be attachments or can contain voice and/or fax.

Each of voice, fax, and text have their own distinct characteristics, which are intuitive to the user. For example, each of these message types require a different media viewer (text editor for text, audio player for voice, and image viewer for fax), and the dimensions of message size are also different for all three (kilobytes for text, seconds for voice, and pages for fax). As a result, a message that includes more than one of these in its parts is termed a mixed media message.

How the messaging client responds to, and acts on these differences is termed "Client Behaviour". This is dependent on the concept of "Message-Context" [2] (previously called primary content), which defines whether the message is a voice mail, fax, or text message. The client can utilize this header to determine the appropriate client behaviour for a particular message.

Traditionally, a messaging "client" referred to some sort of visual interface (or GUI - graphical user interface) that was presented on the users computer. However, as messaging evolves to unified communications the actual form of the messaging client is expected to change. Today's email can often be viewed on wireless devices with very limited screens or even "viewed" over a telephone (i.e., listening to email as you would listen to voice mail through a TUI telset user interface).

The intent of this document is to be general and refer to all types of messaging clients, as the user's expectation of behaviour based on the type of message is not expected to change. However, some of the following concepts may tend towards the more common GUI client.

\section{Conventions Used in This Document}

In examples, "C:" and "S:" indicate lines sent by the client and server respectively.

The key words "MUST", "MUST NOT", "REQUIRED", "SHALL", "SHALL NOT", "SHOULD", "SHOULD NOT", "RECOMMENDED", "MAY", and "OPTIONAL" in this document are to be interpreted as described in RFC-2119 [4] . 


\section{Message Icon}

The preferred method to distinguish between voice, fax, and text messages on a GUI client is with a visual cue, or icon. A similar voice prompt or "earcon" would be used for TUI clients.

As it is possible for the message to contain more than one media type, the icon should describe the primary message content, as defined by the "Message-Context" header. Obvious choices for the icon/message pairs would be a telephone for a voice message, a fax machine for a fax message, and an envelope for a text mail message. Similarly obvious for the earcons would be short spoken prompts like "voice message".

This could be taken a step further, and have the GUI icon change to indicate that the message has been read as is currently done in some email clients (others do not change the icon but merely bold the message in the message list to indicate it is unread). For example, a telephone with the receiver off-hook could indicate that the voice message has been played. A fax machine with paper at the bottom, as opposed to the top, would show that the fax had been viewed. Finally, an open envelope indicates that a text message has been read.

\subsection{Proposed Mechanism}

As the choice of icon is determined by the primary message type, the client should obtain this information from the "Message-Context " message header. This header is defined in [2].

4. Sender's Number Column

As is the case with most email GUI clients today, important message information is organized into columns when presented to the user in a the summary message list. TUIs often present even briefer summaries to the user at the beginning of the session. Typical columns in the GUI client include the message subject, and the date the message was received.

Another important piece of information for the user is the origin of the message. For a voice or fax message, the origin is typically a telephone or fax machine respectively, each of which has an associated telephone number. This telephone number is critical to the user if they wish to return the call. This should be presented accurately to the user (without making it an email address). 


\subsection{Proposed Mechanism}

Instead of forcing the telephone number into an email address, a new Internet message header can be used to hold the originating telephone number [3]. If the message is indicated as being a voice or fax message per [2], the client should extract the number, and display it to the user in a separate column. As this header is defined to only hold the digits of the telephone number, it is left to the client to add any separating characters (e.g., "-") .

5. Message Size

In the cases of large attachments, small clients (e.g., PDA) and slow links (e.g., wireless) there is also a need for the client to see the length of the message in a suitable format before opening it.

Currently, message size is normally given in kilobytes (kB). This is sufficient for plain text messages, but while it may give a hint as to how good the compression algorithm is, $\mathrm{kB}$ is not very useful in knowing the size of a voice and/or fax message. Instead, the size should give an indication of the length of the message, i.e., the duration (in seconds) of a voice message, and the number of pages of a fax. Again, the message may contain multiple types, so the size displayed should be that of the primary content type, per [2].

\subsection{Proposed Mechanisms}

There are three suggested methods to relay this information, of them, the first method is favored:

5.1.1. MIME Header Content-Duration as described in RFC 2424 [5]

For voice messages, the Content-Duration field of the main audio/* body part (as indicated by content-disposition per [1]) should be displayed as the length of the message. If there are several audio parts, an implementation may display the message size as an aggregate of the length of each.

For fax messages a new MIME Header, Content-Page-Length, could be defined, similar to Content-Duration with the exception that number of pages would be specified, rather than number of seconds. (e.g., Content-Page-Length:3). This would be created at origination. 
5.1.2. Message length indicated as a parameter of an Existing RFC 2045 [7] Content-Type Header Field

This would be created at the source. This proposed method would allow the message length to be passed to the client by default in IMAP. Again the client would have to choose between the main voice message length or an aggregate message length for display.

Content-Type Header Field example:

Content - Type=audio/*; length $=50$

Content-Type=image/tiff; pages $=3$

5.1.3. Message length indicated as part of an existing RFC 2822 [9] Header Field

This field would be created at the source and may include message length information, but because it is part of the message headers, it could also be amended on reception (by a local process). This method would allow the message length to be passed to any client by default and not require any client modification. If used, this field would indicate the aggregate length of all attachments.

The advantage of this mechanism is that no new headers are required and it works with existing clients. The downside is that it overloads the subject field.

Subject Header Field example:

Subject $=$ Voice Message (0:04)

Subject $=$ Fax Message (3p)

Subject $=$ Voice Message $(0: 14)$ with Fax (1p)

6. Media Viewer

When a message is initially opened, the client should, by default, open the proper media viewer to display the primary message content. That is, an audio player for voice messages, an image viewer for fax, and a text editor for text messages. Note that on a TUI, the viewer would render the media to sound (which would have varying effect depending on the media and available process).

Where there is more than one body part, obviously the appropriate viewer should be used depending on which body part the user has selected. 
In the case where several viewers are available for a single media type, the user should be prompted to select the desired viewer on the first occasion that the message type is encountered. That viewer should then become the default viewer for that media type. The user should have the ability to change the default viewer for a media type at any time.

Note that it is possible that the media viewer may not be part of the client or local to the host of the client. For example, a user could select to play a voice message from a GUI and the message is played over a telephone (perhaps because the user has no desktop speakers). Additionally, a user listening to a unified messaging inbox over a TUI could chose to print a particular message to a nearby fax machine.

\subsection{Proposed Mechanism}

As mentioned, the default viewer displayed to the user should be the appropriate one for the primary message type. The client is able to determine the primary message type from the "Message-Context" message header per [2].

7. Mark Message as Read

Obviously, the user must be able to know which messages they have read, and which are unread. This feature would also control the message icon or earcon as mentioned in section 1 .

With the proliferation of voice and fax messages, clients should only indicate that these messages are read when the primary body part has been read. For example, a voice message should not be indicated as read until the audio part has been played. The default is currently to mark a message read, when the first body part (typically text) is viewed.

7.1. Proposed Mechanism

Implementation of this feature on most clients is a local issue.

For example, in the case of IMAP4 [6], these clients should only set the \SEEN flag after the first attachment of the primary content type has been opened. That is, if the message context is voice message, the ISEEN flag would be set after the primary voice message (indicated by content-disposition [1] or content-criticality [8]) is opened. 


\section{Security Considerations}

The desirable client behaviours described here are intended to provide the user with a better client experience. However, supporting the proposed behaviours described in this document does not make a client immune from the risks of being a mail client. That is, the client is not responsible for the format of the message received, it only interprets. As a result, messages could be spoofed or masqueraded to look like a message they are not to elicit a desired client behaviour. This could be used to fool the end user, for example, into thinking a message was a voice message (because of the icon) when it was not.

9. Informative References

[1] Vaudreuil, G. and G. Parsons, "Voice Profile for Internet Mail version 2 (VPIMv2)", RFC 3801, June 2004.

[2] Burger, E., Candell, E., Eliot, C., and G. Klyne, "Message Context for Internet Mail", RFC 3458, January 2003.

[3] Parsons, G. and J. Maruszak, "Calling Line Identification for Voice Mail Messages", RFC 3939, December 2004.

[4] Bradner, S., "Key words for use in RFCs to Indicate Requirement Levels", BCP 14, RFC 2119, March 1997.

[5] Vaudreuil, G. and G. Parsons, "Content Duration MIME Header Definition", RFC 3803, June 2004.

[6] Crispin, M., "INTERNET MESSAGE ACCESS PROTOCOL - VERSION 4rev1", RFC 3501, March 2003.

[7] Freed, N. and N. Borenstein, "Multipurpose Internet Mail Extensions (MIME) Part One: Format of Internet Message Bodies", RFC 2045, November 1996.

[8] Burger, E., "Critical Content Multi-purpose Internet Mail Extensions (MIME) Parameter", RFC 3459, January 2003.

[9] Resnick, P., "Internet Message Format", RFC 2822, April 2001.

[10] Parsons, G., "IMAP Voice Extensions", Work in Progress, June 1999 . 


\section{Acknowledgments}

This work was inspired by the discussion of "Proposed Mechanisms" for IMAP that were detailed in a since expired work in progress entitled "IMAP Voice Extensions" [10]. The authors would like to acknowledge all those who contributed to that document. In addition, Cheryl Kinden, Derrick Dunne, and Jason Collins assisted in the editing of previous revisions of this document.

Author's Addresses

Glenn Parsons

Nortel Networks

P.O. Box 3511, Station C

Ottawa, ON K1Y $4 \mathrm{H} 7$

Canada

Phone: +1-613-763-7582

Fax: +1-613-967-5060

EMail: gparsonsanortel.com

Janusz Maruszak

Phone: +1-416-885-0221

EMail: jjmaruszakesympatico.ca 
Full Copyright statement

Copyright (C) The Internet Society (2005).

This document is subject to the rights, licenses and restrictions contained in BCP 78, and except as set forth therein, the authors retain all their rights.

This document and the information contained herein are provided on an "AS IS" basis and THE CONTRIBUTOR, THE ORGANIZATION HE/SHE REPRESENTS OR IS SPONSORED BY (IF ANY), THE INTERNET SOCIETY AND THE INTERNET ENGINEERING TASK FORCE DISCLAIM ALL WARRANTIES, EXPRESS OR IMPLIED, INCLUDING BUT NOT LIMITED TO ANY WARRANTY THAT THE USE OF THE INFORMATION HEREIN WILL NOT INFRINGE ANY RIGHTS OR ANY IMPLIED WARRANTIES OF MERCHANTABILITY OR FITNESS FOR A PARTICULAR PURPOSE.

Intellectual Property

The IETF takes no position regarding the validity or scope of any Intellectual Property Rights or other rights that might be claimed to pertain to the implementation or use of the technology described in this document or the extent to which any license under such rights might or might not be available; nor does it represent that it has made any independent effort to identify any such rights. Information on the procedures with respect to rights in RFC documents can be found in BCP 78 and BCP 79 .

Copies of IPR disclosures made to the IETF Secretariat and any assurances of licenses to be made available, or the result of an attempt made to obtain a general license or permission for the use of such proprietary rights by implementers or users of this specification can be obtained from the IETF on-line IPR repository at http://www.ietf.org/ipr.

The IETF invites any interested party to bring to its attention any copyrights, patents or patent applications, or other proprietary rights that may cover technology that may be required to implement this standard. Please address the information to the IETF at ietfipreietf.org.

Acknowledgement

Funding for the RFC Editor function is currently provided by the Internet Society. 